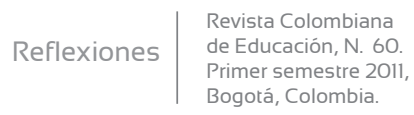

\section{La tardía instalación de la teoría del desarrollo cognitivo de Jean Piaget en Colombia, 1968 - 2006}

//Belated Implementation in Colombia of Jean Piaget's Theory of Cognitive Development, 1968 - 2006 $\begin{array}{ll} & \text { Recibido: 14-ene-11 } \\ & \text { Evaluado: 3-may-11 } \\ \text { Absalón Jiménez* } & \text { Arbitrado: 20/06/11 }\end{array}$

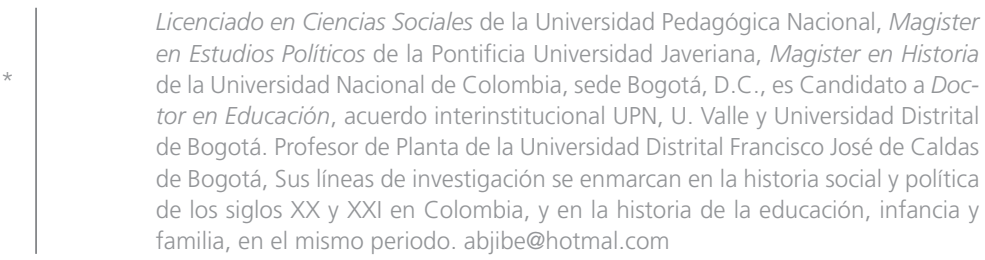

\section{Resumen}

El presente artículo da a conocer la manera como la teoría del desarrollo cognitivo de Jean Piaget, se instala en Colombia de manera tardía en las últimas décadas del siglo XX e inicios del XXI. La divulgación final de la obra de Piaget en Colombia, particularmente la teoría del desarrollo cognitivo, debe ser valorada bajo una serie de condiciones de posibilidad, entre ellas se destacan: en primer lugar, la traducción de las obras de Jean Piaget a la lengua castellana, solo hasta la primera mitad de la década de los años setenta, lo que permitió que este autor dejara de ser monopolio académico de unos pocos intelectuales colombianos, para masificar su teoría en los centros de formación de docentes en el país; en segundo lugar, la masificación del preescolar en Colombia, lo que demandó la constitución de un estatuto para la primera infancia, en el cual la teoría del desarrollo cognitivo jugaría un papel fundamental; y en tercer lugar, la labor difusora de la teoría del desarrollo por parte del Instituto Colombiano de Bienestar Familiar (ICBF), fundado en 1968, el Ministerio de Educación y la Unicef, con el fin de transformar de manera directa dos prácticas sociales: las educativas escolares y las de crianza.

\footnotetext{
Abstract

This paper presents how Jean Piaget's cognitive development theory is implemented belatedly in Colombia during last decades of 20th century and beginning of 21 st century. Piaget's work final dissemination in Colombia Final Piaget's work dissemination in Colombia, especially his cognitive development theory should be evaluated under certain possibility conditions, among them: firstly, the translation into Spanish of Jean Piaget's work until the first half of 1970 's, so this author stopped being read by a few Colombian intellectuals for giving access to his theory to the teacher training institutions of the country; secondly, preschool education spread in Colombia, which required the establishment of a charter for early childhood where the cognitive development theory would play a critical role; and finally, the diffusion labor of the development theory, motivated by the Instituto Colombiano de Bienestar Familiar-ICBF- founded in 1968, the Ministry of Education and Unicef, in order to transform directly two social practices, i.e. school educational and parenting practices.
}

\section{Palabras Clave}

Infancia, desarrollo cognitivo, Pedagogía, Educación, crianza.

\section{Keywords}

Childhood, cognitive development, pedagogy, education, parenting. 


\section{Introducción}

La teoría del desarrollo cognitivo fundada a mediados del siglo XX por Jean Piaget, ha tenido un enorme impacto en la Educación, tanto en lo que respecta a las elaboraciones teóricas como a los modelos y práctica pedagógica. Actualmente, el uso y el aporte de la teoría de Piaget se ubica dentro de cierto tipo de modelo constructivista en Educación y, en una serie de sub-modelos de innovación pedagógica, en el que el conocimiento y el aprendizaje no constituyen una copia de la realidad, sino una construcción activa del sujeto en interacción con su entorno. Desde la teoría del desarrollo cognitivo se reconoce a un sujeto activo, que se constituye en un escenario intersubjetivo y cambia de manera cualitativa de acuerdo a un proceso de socialización y educación en el que incide de manera directa el entorno socio cultural.

Es nuestro interés particular, en el presente artículo dar a conocer la tardía instalación de la teoría de desarrollo cognitivo de Jean Piaget en Colombia, como también valorarla como formación discursiva que incidiría en dos tipos de práctica en particular: en primer lugar, en la práctica escolar, en el devenir del preescolar en Colombia, en las últimas décadas del siglo XX e inicios del XXI, en el que la primera infancia logra un estatuto importante en la escuela; en segundo lugar, en las prácticas de crianza familiar, en las que la teoría del desarrollo se convierte en un eje fundamental, con el fin de educar a la familia teniendo en cuenta los cambios de carácter cualitativo y de desarrollo en el niño.

Como se observará en el presente escrito, no es nuestro interés desarrollar una discusión teórica o conceptual en torno a la obra de Jean Piaget. Más bien nuestro interés es el de reconstruir un discurso y dar cuenta de la manera como la teoría del desarrollo se instala en nuestro país en el ámbito académico y social de manera tardía, más bien hacia las últimas décadas del siglo XX e inicios del XXI. Para tal efecto, rastreamos la presencia de Jean Piaget y su teoría en el diario El Tiempo, entre 1968 y 2006, periodo en el cual evidenciamos la reseña de dos de sus obras, en 1972, las cuales se centran en el problema escolar, Psicología y Pedagogía y A dónde va la Educación.

Es importante resaltar que solo hacia la primera mitad de los setenta se dan a conocer las primeras traducciones al castellano de las obras de este destacado psicólogo, lo que permitió que este autor dejara de ser monopolio académico de unos pocos intelectuales colombianos, para masificar su teoría en los cen- 
tros de formación de docentes en el país. Luego, a lo largo del rastreo, evidenciamos cómo el Instituto Colombiano de Bienestar Familia, ICBF, fundado en 1968, el Ministerio de Educación Nacional y la Unicef, se encargan de dinamizar la teoría del desarrollo como eje fundamental en la práctica escolar y la práctica de crianza. A largo del presente escrito, con base en un ejercicio arqueológico, logramos comprobar, entre otros aspectos, que solo a finales del siglo $\mathrm{XX}$, la teoría del desarrollo de Jean Piaget se instala de manera masiva y definitiva. Dicha teoría en nuestro país ha sido fundamental para darle cierto estatus a la infancia, de manera particular a la primera infancia; es decir, la comprendida entre los 0 y 6 años de edad. No obstante, la teoría del desarrollo cognitivo, es valorada por una serie de prácticas sociales que recogen conceptos piagetianos, los cuales son complementados e integrados con otra serie de aportes, provenientes de los enfoques pedagógico, sociológico y moral.

En este sentido, parodiado a Paul Veyne, hay que ubicar los cambios con relación al sujeto en "la transformación de las prácticas" (Veyne, 1984, p. 199); ${ }^{1}$ es decir, en este caso en particular preguntarnos, ¿cuáles son las prácticas que objetivan a la infancia y cómo se han transformado? Es este sentido, en primer lugar

Para este historiador, las representaciones y los enunciados forman parte de la práctica, de tal manera podríamos pensar que la infancia no es un producto dado, sino es parte del constructo de una práctica. nos preguntarnos por la transformación de las prácticas en torno a la educación del niño y su aprendizaje en la escuela. En segundo lugar, las transformaciones en el cuidado familiar y las prácticas de crianza. Creemos que en la transformación de estas dos prácticas la teoría del desarrollo cognitivo e infantil jugó un papel fundamental como dinamizador de una serie de transformaciones discursivas y sociales.

En el marco del presente escrito creemos que la teoría del desarrollo cognitivo de Jean de Piaget, representa uno de los aportes más importantes de la historia de la psicología. El desarrollo cognitivo es entendido como una sucesión de cambios discontinuos o estadios, en los cuales el sujeto va aumentando en capacidad explicativa, pensamiento formal y conquista de autonomía. La teoría del desarrollo vista como una formación discursiva, la podemos asumir como un conjunto de reglas anónimas, históricas siempre determinadas en el tiempo y en el espacio, que han definido en una época dada y para un área social en particular, las condiciones de ejercicio de una función enunciativa, en este caso, en torno a la infancia. Enunciados tales como: desarrollo, aprendizaje, asimilación, acomodación y socialización, determinan la disposición de un particular tipo de infancia que pensada en la modernidad, vive continuamente un cambio de carácter cualitativo. El anterior régimen de enunciados, constituye 
un conjunto de configuraciones y relaciones discursivas que un enunciado cualquiera puede adoptar en un momento determinado con respecto a otro, ya sea en el plano de la sucesión o la simultaneidad.

\section{La teoría del desarrollo infantil en la práctica escolar y la primera infancia}

A finales de los años sesenta, los especialistas y educadores se preguntaban, ¿por qué solamente a los seis años y medio era que el niño debía ingresar a primero de primaria?, siendo este el curso en que se dictaban las clases de lectura y escritura. Mientras tanto, el niño, entre los dos y seis años y medio, cantaba, jugaba, corría, tocaba instrumentos, caminaba, saltaba, Iloraba y hasta reía. Las transformaciones de la sociedad colombiana demandaban la instauración de un nuevo acuerdo, un nuevo dispositivo de alianza entre la familia y la escuela, pero ya no pensada para la segunda infancia; es decir, el grupo de niños que se ubican entre los siete y doce años de edad, el cual había sido objeto de un proceso paulatino de objetivación por parte de la Pedagogía colombiana sin reparar a fondo el tema de la primera infancia, el grupo de niños ubicados entre los cero y seis años.

Para finales de los años sesenta e inicios de los setenta, la prioridad comenzaba a ser la primera infancia, que como concepto y categoría viviría un proceso de objetivación en el cual la teoría del desarrollo infantil, de Jean Piaget, jugarían un papel fundamental. Al realizar un rastreo arqueológico de la primera superficie de emergencia enunciativa de este discurso, observamos como hacia 1968 el aumento de las instituciones de preescolar en todo el mundo daba cuenta de la relación que la sociedad re-establecía con este tipo de instituciones encargadas de la primera infancia ${ }^{2}$. El preescolar, en su devenir como un tipo de escuela particular, se constituyó como forma de socialización privilegiada y lugar de

2 En Colombia para 1960, los establecimientos de preescolar apenas llegan a los 974, los cuáles solo satisfacían el $1 \%$ de las necesidades de la población en edad preescolar. Por los demás, para este año apenas 29 eran oficiales y 945 pertenecían al sector privado. Luego, para 1965, hay 1.099 instituciones de preescolar, 49 oficiales y 1.050 privadas; en 1975, encontramos 1.837 instituciones 502 oficiales y 1.335 privadas; en 1980, 3.281 instituciones de preescolar, 1.302 oficiales y 1.979 del sector privado; y en 1983, de 4.396 instituciones, 2.084 pertenecían al sector oficial y 2.312 al sector privado. Desde 1960 se paso a tener un número aproximado de 40.000 niños matriculados en preescolar, a 240.000 en 1974, antes de comenzar a funcionar los "Centros de atención integral de preescolar" CAIP, perteneciente al ICBF, fecha en que se masifica el preescolar en Colombia (Consultar: Hugo Cerda, La institución preescolar, U. Santo Tomas, Bogotá, D.C., 1986, p. 87). 
paso obligatorio para los niños de las clases populares. El preescolar en Colombia, terminó de constituir una maquinaria particular de gobierno sobre la infancia, que demandó el establecimiento de un espacio específico, que conllevó la delimitación de unos tiempos de infancia, como lo fue en su etapa inicial y, bajo un sentido práctico en el ámbito nacional, la sala-cuna, entre los cero y dos años; la guardería, entre los dos y cuatro años, y el jardín, entre los cuatro y seis años.

La educación preescolar devino así, a finales de los años sesenta y comienzos de los setenta, de las preocupaciones en torno a la formación del niño. La familia demandaba una entidad colaboradora que no perjudicase o interfiriese sus funciones fundamentales. A las primeras instituciones que tenían una función de guardería, se les exigió tres grandes finalidades: protección, formación y cuidado. Dichas instituciones, que poseían un saber en cuanto formas de domesticación del sujeto, terminaron colaborándole a la familia en el proceso de socialización y educación de la primera infancia.

Desde inicios de los años setenta el devenir del preescolar en Colombia, estuvo acompañado, de manera explicita, de la teoría del desarrollo infantil, establecida por Jean Piaget, la cual sería re-leída y re-valorada en el contexto de una nueva época en la que se institucionalizó de una vez por todas, la educación preescolar como política en el ámbito nacional masificándose como propuesta. Las teorías del desarrollo infantil incidirían de manera directa en el proceso de "psicologización" y "pedagogización" del niño que se vivió en Colombia en las últimas décadas del siglo XX. De tal manera, evidenciamos cómo para el año 1972, se reseñaba por primera vez del castellano, y no por medio de una traducción, la obra de Jean Piaget, de quien se aclaraba que era doctor en Ciencias Naturales y una de las autoridades más conocidas de la Psicología y la Pedagogía. Piaget, como psicólogo del desarrollo de la inteligencia, sería re-leído en el contexto de los años setenta, pero acompañado de nuevas demandas producto del proceso de masificación paulatina que viviría el preescolar como propuesta. Uno de los aspectos fundamentales de las teorías del desarrollo era valorar la relación que el niño establecía con su medio, debido a que para Piaget:

El organismo altera el medio y lo hace en función de sí mismo, aspecto que es definido como asimilación; por otra parte, el organismo se altera en función del medio, aspecto que es definido como acomodación. Todo acto de acomodación al medio tiene algo de los dos aspectos. Como la nutrición, el conocimiento es un proceso adaptativo y supone una modificación a nivel del intelecto, ya del medio en beneficio suyo (asimilación), ya de sí 
mismo en beneficio del medio (acomodación). La asimilación intelectual se produce cuando el sujeto se enfrenta a un objeto ambiental, lo estructura o reestructura con base a la organización intelectual que le es propia ${ }^{3}$.

Se le aclaraba a los especialistas colombianos que, para Piaget, todo acto intelectual por sencillo que pareciera, suponía una interpretación de la realidad externa; es decir, que siempre habría una asimilación del objeto. De tal manera, como el sujeto tiene ya una serie de esquemas, una organización intelectual dada, a ella se integra el objeto nuevo. De acuerdo a la teoría de desarrollo infantil de Piaget, la inteligencia se desarrolla desde lo puramente lógico hasta lo más abstracto, reconociéndose tres periodos sucesivos:

a. Periodo de la inteligencia sensomotora. Se inicia con una completa indiferenciación del yo y del mundo exterior y termina cuando el niño adquiere una organización relativamente coherente de las acciones sensorio-motoras entre su ambiente inmediato. Esa coherencia y su organización son prácticas sin conceptualización alguna.

b. Periodo de las operaciones concretas. Se inicia con simbolizaciones elementales que se presentan al final del periodo anterior, y concluye con los comienzos del pensamiento formal durante los primeros años de la adolescencia.

c. Periodo de las operaciones formales. Se produce una reorganización definitiva, con nuevas estructuras. El adolescente puede enfrentarse no solo a la experiencia concreta directa, sino a problemas y realidades que exijan una mayor abstracción $n^{4}$.

El conocimiento por parte de los pedagogos colombianos, de los niveles intelectuales a que es capaz el niño, haría más efi-

3 "Psicología Pedagógica: Piaget, la inteligencia infantil". El Tiempo, 25 de enero de 1972, p. 12C. Es de anotar que la prensa, desde los años setenta, incide en la difusión de la teoría del desarrollo cognitivo haciendo mención a las obras de Jean Piaget, dedicadas exclusivamente a problemas educativos, las cuales son: J. Piaget, Psicología y pedagogía, Ariel. Barcelona, 1973; y J. Piaget, A dónde va la educación, Teide, Barcelona, 1974. Estas obras representan su primera transcripción masiva al español, aspecto fundamental para su difusión en nuestro país.

4 Ibid. 
ciente y económica la enseñanza. En este sentido, los profesores solían cometer errores al olvidar la importancia de la acción en el desarrollo de la mente. O a nivel de la enseñanza primaria y aún del preescolar, la tendencia a teorizar demasiado sin tener en cuenta que tal exigencia de nada servía, si no se trasmitía el conocimiento proporcional a la edad del niño por la acción. Otro elemento importante, era no subestimar la capacidad de abstracción del niño.

El preescolar, como institución y el aula como espacio, terminó de constituir el aislamiento necesario del niño con relación a la familia, a la vez que el propio concepto de infancia quedó asociado a la formación casi natural de la demarcación espacio temporal. El aula implicó la ejecución de una estructura de comunicación entre sujetos; estaba definida tanto por la arquitectura y el mobiliario escolar, como por las relaciones de autoridad, comunicación y jerarquía que aparecían en el aula, tal como la conocemos, y que eran básicas a la hora de enseñar que muchas veces pasaban inadvertidas (Dusel y Caruso: 2006: 31). La separación de los niños del preescolar por edades, espacios particulares y grados de desarrollo, fueron fundamentales, acompañados del aula como un elemento clave en la complementación del dispositivo.

Para la pedagoga Olga Sarasti, en 1972, la etapa comprendida entre los dos y medio y seis años, había sido Ilamada la edad olvidada de la niñez:
Es cuando el pequeño aprende a caminar, hablar, pelear, pero aún no está capacitado para asistir a una escuela o colegio. ¿Qué pasa entre los dos años y medio y los seis?, edad en la que se menosprecia el desarrollo del niño. ¿Por qué nadie se fija en ellos y al contrario empiezan a molestar y a estorbar a la mamá? Los padres olvidan que la etapa más importante de la vida de un ser humano es precisamente la infancia y más específicamente esta edad, cuando el pequeño necesita ampliar sus conocimientos, quiere saber de qué están hechas las cosas, cómo funcionan, qué tienen por dentro, etc. Los jardines infantiles son lugares donde se define el $80 \%$ de la personalidad del pequeño ${ }^{5}$.

La infancia genera un campo de conocimiento que la Pedagogía construye, pero a la vez es un cuerpo, el cuerpo infantil, depositario del accionar específico de la educación escolar. Ser alumno en una institución escolar consistía en ocupar un espacio de inscripción de saberes y poderes; se concebía igualmente al cuerpo como inerme, que debía ser formado, disciplinado, educado, en función de una utopía sociopolítica, la educación para todos y una metodología universal. La capacidad del niño, en términos cognitivos y de su edad, representaba dos elementos clave para la distribución del cuerpo infantil en la escuela de allí la im-

5 "Los jardines infantiles no son un juego", El Tiempo, 20 de diciembre de 1972, p. 3B. 
portancia de la teoría del desarrollo infantil en momentos en que se masificaba el preescolar en Colombia.

Para Mariano Narodowski, en este periodo era imposible comprender el proceso de construcción de la infancia moderna sin considerar el discurso pedagógico, acompañado del discurso de la Psicología del niño y el de la pediatría, como operador y dador de sentidos acerca de la infancia. La institución escolar moderna es el dispositivo que se construye para encerrar a la niñez. Encerrarla, tanto desde el punto de vista topológico o corpóreo (encierro material) como también desde las categorías que la Pedagogía ha elaborado para construirlas (encierro epistémico), (Narodowski,: 1991:91). La irrupción masiva del preescolar, en las últimas décadas del siglo $\mathrm{XX}$, se constituye en un nuevo dispositivo en el que el objeto de poder y saber, es la infancia, pero en su más tierna edad, entre los cero y seis años de edad, la cual demanda un nuevo encierro físico y topológico como también un encierro epistémico en el que la teoría del desarrollo de Piaget, jugaría un papel fundamental.

En este sentido, para la Unicef, a comienzos de los años ochenta, los programas de educación infantil temprana, se habían convertido en una preocupación de los gobiernos de la región, pasándose del "paradigma de la beneficencia y asistencia social" de los años sesenta, "al paradigma del desarrollo infantil". Dicho tránsito se había dado en momentos en que se comenzaba a pasar de la familia extensa a la familia nuclear: "Lo que había significado un menor número de familiares disponibles para cuidar de los niños. Ya las madres no podían contar con las abuelas y las tías, que podían estar también trabajando. La fuerte migración también había agravado el aislamiento de la familia y en muchos casos, había implicado que las mujeres se hubieran convertido en jefes de hogar" ${ }^{\prime \prime}$.

La Unicef, en 1981, se pronunció en torno a esta doble tensión, la cual evidenciaba un doble modelo de la Educación preescolar. En primer lugar, el modelo de beneficencia y cuidado a los niños pobres, hijos de la clase trabajadora, modelo mediante el cual había masificado el preescolar en Colombia en los años setenta; y en segundo lugar, el modelo propio del "desarrollo cognitivo", mediante el cual se viabilizarían las propuestas educativas para la primera infancia desde los años ochenta. Así 
para la Unicef, existían dos vertientes principales:

En la primera, le atribuye mayor importancia a los ingresos familiares y a la atención infantil, para que permitiera a los miembros de la familia, especialmente a las mujeres, trabajar por fuera del hogar. En la segunda variante, el énfasis se coloca sobre el desarrollo del niño bajo la presunción de que las mejoras en los años tempranos no solamente aumentarán las oportunidades de que los niños pobres desarrollen su potencial como miembros productivos de la sociedad, sino también podrán seguramente mantenerse al ritmo de los niños aventajados a medida que aprenden y devengan en su vida posterior ${ }^{7}$.

El interés de la Unicef, desde los años ochenta, fue el de no desconocer los dos tipos de realidades expresadas en las dos vertientes. La primera, por presión social, y la segunda, como parte de un paradigma modernizante que permitía diferenciar al niño en etapa de preescolar cualitativamente. En general, el establecimiento del preescolar como nuevo dispositivo de la alianza, entre la familia y la escuela, delimitaba, rodeaba y controlaba al niño en su primera infancia. Ya fuese por razones sociales, económicas, psicológicas, pedagógicas y pediátricas, se daba cuenta por medio de una serie de discursos, de un nuevo tipo de encerramiento, tanto físico como epistemológico, de la infancia en su primera etapa de desarrollo. Una de las importancias de esta nueva alianza era la posibilidad que brindaba para pensar a la infancia como categoría que se transforma en las últimas décadas del siglo $X X$ e inicios del XXI, reconociendo al niño escolarizado desde su más tierna edad como sujeto, al cual el padre de familia, el maestro y la escuela debía valorar como tal.

A mediados de los años noventa la Unicef y el ICBF, dieron a conocer la Escala de valoración cualitativa del desarrollo infantil, desde el nacimiento hasta los seis años ${ }^{8}$. Dicha escala tenía como objetivo fundamental explicitar la importancia de la teoría de desarrollo, incidiendo en los proceso de crianza y educación de la primera infancia en Colombia, con el fin de crear las condiciones para que se hiciere posible el despliegue de la vida infantil y el desarrollo psicológico del niño. Para estas instituciones, al adulto, y particularmente al docente, le correspondía comprender su papel en la conformación del psiquismo del niño y su evolución. La escala de valoración cualitativa del desarrollo infantil del niño en Colombia, partía del objeto conceptual de la evaluación, el mismo desarrollo infantil del sujeto. Mediante este se preten-

8 Unicef -ICBF, Escala de valoración cualitativa del desarrollo infantil, desde el nacimiento hasta los seis años, Bogotá, D.C., 1995. 
día dar cuenta del grado de desarrollo psicológico de los niños menores de seis años en sus diferentes dimensiones.

Para la Unicef y el ICBF, el desarrollo infantil debía hacer énfasis en un modelo integrador de los procesos psicológicos, en el cual se integraban los componentes motores, cognitivos-representativos, afectivos y sociales. La conceptualización del desarrollo del ICBF, se ubicaba dentro del planteamiento de modelos interaccionistas del desarrollo, de Jean Piaget, acompañado de los aportes de Jerome Bruner y Liev Vygotsky, dándole énfasis a los procesos socioculturales dentro de los cuales se desarrollaba el niño. El desarrollo infantil propuesto por estas instituciones partía de conocer que los procesos subjetivos y psicológicos, aunque diferentes, son indisociables del contexto sociocultural en el cual se originaban. Comprender el nivel de desarrollo alcanzado por el niño, requería tener en consideración el contexto de socialización y, en particular, la calidad de las relaciones que le ofrecían los adultos responsables de la Educación de los niños. Dentro del proceso de desarrollo infantil, la propuesta de la Unicef y del ICBF, se diferenciaban cuatro etapas: primera edad (de cero a un año); infancia temprana (entre uno y tres años); edad preescolar (entre los tres y cinco años); y transición (seis años), cada una de ellas caracterizada por el tipo de relación que el niño establece consigo mismo, con los demás y con el mundo que los rodea.

El preescolar, como institución, delimitó a la infancia contemporánea escolarizada en un doble sentido, tanto de desarrollo cognitivo como también al convertirse en un elemento clave en la preparación del niño para la escuela primaria. El preescolar participó finalmente del proceso de "alumnización" y escolarización que vivió el niño en esta nueva institución. En las últimas décadas del siglo XX, el plan de estudios para la educación preescolar propendió por el desarrollo integral del niño en sus aspectos biológico, sensomotor, cognitivo y socio afectivo, contribuyendo así a su desarrollo armónico e integral por medio de la lúdica y el juego. El crecimiento armónico y equilibrado del niño, constituyó una preocupación de la escuela como institución, el desarrollo de su creatividad, las habilidades y destrezas propias de la edad, la ubicación espacio temporal y el ejercicio de la memoria, además del desarrollo del lenguaje y la comunicación.

La objetivación de la infancia en Colombia tiene un punto de llegada importante en los Lineamientos Curriculares de Prees- 
colar, dados a conocer en el ámbito nacional en 1998, documento en el cual se recoge la discusión que venía dándose, por lo menos, desde finales de años sesenta por parte del ICBF, la Unicef, el Ministerio de Educación y el sector educativo en general, en torno a una infancia que debía ser vista de manera integral como sujeto, desde la perspectiva tanto de desarrollo cognitivo, desarrollo moral, socio afectiva y de desarrollo humano, bajo la guía de un proyecto lúdico pedagógico, que reconoce a la infancia como un sujeto cognoscente y como un sujeto que conquista, de manera paulatina, no solo conocimiento, sino también una identidad; es decir, que se constituye una subjetividad.

Como formación discursiva la teoría del desarrollo cognitivo instala en la primera superficie de emergencia una serie de enunciados fundamentales, tales como: desarrollo, aprendizaje, esquema, asimilación y acomodación. Los anteriores terminan de articularse en el ámbito social con otra serie, como: primera infancia, preescolar, armonía equilibrio, creatividad, lúdica, juego y educación. Dichos enunciados de manera paulatina organizan una nueva realidad en torno a los temas de infancia en nuestro país, incidiendo en el ámbito discursivo y no discursivo. Así mismo, se constituye como principal instancia de delimitación una teoría en particular, la teoría de desarrollo cognitivo, la cual comenzaría a ser estudiada de manera sistemática por una serie de profesionales, entre ellos, los psicólogos y los pedagogos; y como principal rejilla de especificación terminan de legitimarse, ante todo, la psicología y la pedagogía, que como disciplinas objetivaban continuamente a la infancia en nuestro país.

\section{La teoría del desarrollo infantil en las prácticas de crianza}

En Colombia, mediante la Ley 75 de 1968, conocida como también la Ley de paternidad responsable, es que nace el Instituto Colombiano de Bienestar Familiar, ICBF, el cual asume las funciones del Consejo Colombiano de Protección Social del Menor y de la Familia y la División de Menores del Ministerio de Justicia. El objetivo del ICBF, desde su inicio, consistió en promover la integración armónica de la familia, proteger las y los menores de edad y garantizar los derechos de la niñez, además, de coordinar las entidades y vincular personas con competencias en el manejo de los problemas de la familia y los menores. De hecho mediante la Ley 75 de 1968, la cual le da vida al ICBF, se asume el problema de la paternidad responsable como un asunto de Estado, como parte del manejo del desarrollo social, con implicaciones económicas y políticas y como elemento consustancial del control de la población.

Una de las preocupaciones centrales del ICBF, desde su nacimiento, 
fue contribuir a salvar a las nuevas generaciones del abandono y la irresponsabilidad de los padres que desamparaban a sus hijos. Salvar a los niños y oponerse al abandono, representa una señal del direccionamiento "biopolítico" del Estado colombiano9. Se trataba reconfigurar a la familia colombiana, incidiendo en un proceso de "re-familiarización" de la sociedad y el Estado, con el fin de ayudar a formar a las nuevas generaciones con los instrumentos institucionales, acompañados de un conjunto de normas sobre paternidad responsable, la tutela eficaz sobre la infancia, la campaña nutricional, la vigilancia de los grupos comunitarios y de los padres sobre sus niños y la coordinación de muchos proyectos dispersos. El control de la familia, en términos de biopolítica, se convirtió en un instrumento eficaz para el control de la infancia y la población, en general, para su devenir social y para el desarrollo del país.

Desde sus inicios el área de más alta prioridad dentro del ICBF, correspondió al desarrollo biológico, psíquico y social del menor, desde su concepción hasta los siete años de vida, y a la promoción de la familia y la comunidad. Las etapas más importantes de crecimiento y maduración del sistema nervioso y asimilación de información relacionada con el medio exterior, se producían durante los periodos de gestación, lactancia y preescolaridad $^{10}$. Por consiguiente, una acción integral sobre la población más vulnerable, en este periodo de vida, condicionaba la existencia de los recursos humanos que pudieran actuar como factor dinámico de producción y cambio social.

Para comienzos de los años setenta, se decía que los cinco millones de niños y el millón y medio de familias, sujetos potenciales del programa, se encontraban dispersos en todo el territorio nacional y muchas veces eran inaccesibles a los posibles servicios que ofrecía el Estado, mediante las políticas de bienestar familiar. El enfrentamiento de estos obstáculos había exigido formular una estrategia definida en los "Centros Comu-

9 La biopolítica trabaja con la población como problema científico y político. Es una expresión del Estado, de saber, de intervención de saber y poder, en torno a temas como la natalidad y morbilidad, las disfunciones biológicas, los efectos del medioambiente y, finalmente, el control propio de la población. (Consultar: Michel Foucault, Estética, ética y hermenéutica. Obras esenciales, volumen III, Paidós, Barcelona, 1999. Ver capítulo: "El nacimiento de la biopolítica", pp. 209-216).

10 Unicef-Colombia, ICBF, Memorias del Primer Seminario Nacional Intersectorial de Alimentación y Nutrición. Comité Nacional de Políticas sobre Alimentación y Nutrición, Palmira, Valle, diciembre 9-12 de 1973. 
nitarios para la Infancia", que con participación de la comunidad, se establecían con un conjunto de recursos integrados provenientes del sector público y privado, cuyo objetivo era garantizar a los menores de siete años, un desarrollo integral, ejercer acciones educativas sobre la familia y estimular procesos de desarrollo en la comunidad. Para el Estado colombiano las acciones directas sobre la familia se convertían en fundamentales, particularmente, en las áreas de desarrollo biológico y psicosocial del niño, el desarrollo intelectual y su preparación para el ingreso a la escuela. Seis años después de creado el ICBF, en 1974, se calculaba que debían estar funcionando al menos 450 unidades de Centros Comunitarios para la Infancia en todo el país, los cuales se preocupaban por la atención integral del niño y el beneficio general de la población colombiana.

La preocupación biopolítica sobre la familia y la infancia, estaría acompañada de un continuo proceso de individualización del niño que comprometía todo el orden social de la concepción de infancia. El niño en el interior de la familia sería el motivo de reflexiones de todo orden desde la perspectiva de las prácticas de crianza las cuales darían un giro explicito, y de manera paulatina, hacia los aportes propios de la psicología y en particular, a las teorías del desarrollo cognitivo e infantil.

Por su parte Unicef, a finales de los años setenta, entraría en este proceso, en el que se promovía cier- to tipo de trabajo con las familias, con el fin de defender a la infancia. Para este organismo internacional, "el hecho de que los primeros años del hombre representan la base de la definición de su personalidad afectiva, se convertía para el año 1979, en un hecho generalmente aceptado. De ahí la importancia de sensibilizar a los padres en el papel esencial que deberán desempeñar al lado de sus hijos, en todas las etapas de su crecimiento y en particular, en aquellas de la edad preescolar; es decir, entre los cero y seis años"11.

De tal manera, la estrecha relación que se establecía entre la educación de un niño pequeño y la actitud de los padres, evidenciaba el interés de un conjunto de especialistas en temas relacionados con la familia, como también de institutos, como el ICBF y la Unicef, en el que se demandaba la integración de los padres dentro de los programas de educación de los niños. Particularmente, para Unicef, convendría, además, que el sistema escolar desempeñara un papel en la formación de los futuros padres, en el marco del conocimiento de las realidades de la vida cotidiana, como la planificación familiar y la educación se-

\footnotetext{
11 Unicef-Colombia, La formación de los padres de familia. Consideraciones relativas a la programación de asistencia educacional del Unicef, Bogotá, octubre de 1979. Este documento como propuesta, buscaba acercar a los padres de familia a las teorías del desarrollo infantil, desde la etapa prenatal hasta los seis años de edad del niño. Es así como se da a conocer una serie de consejos para contribuir en el desarrollo motriz, las percepciones, el lenguaje y las relaciones con el prójimo.
} 
xual, la economía doméstica y el medio ambiente de carácter social. Partir del clima cultural y afectivo del niño podría generar las condiciones de un desarrollo armonioso en el que no se debían imponer nociones, sino más bien desarrollar aptitudes en pro del bienestar de la familia.

En Colombia, a lo largo de los años ochenta y noventa, se evidencia una preocupación por asistir a las familias pobres, promover la integración armónica de la familia, acompañada de la paternidad responsable, como también el desarrollo de medidas preventivas frente al abandono de la niñez.

Partiendo de los anteriores antecedentes, el ICBF a inicios de los años noventa, promovería de manera explícita, la teoría del desarrollo cognitivo e infantil dentro de las prácticas de crianza familiar. En este sentido, dio a conocer un documento, en 1990, que fue de gran importancia en el proceso de psicologización del niño en el interior de la familia, El desarrollo infantil, una conceptualización desde el ICBF'2, en el que se conceptualizaba el desarrollo infantil, explicando de manera práctica la evolución del desarrollo del niño, desde el nacimiento hasta los seis primeros años de vida, teniendo en cuenta como ámbitos fundamentales: la relación consigo mismo, la relación con el mundo que lo rodea y la relación con los otros. Dicha conceptualización se planteaba como objeto orientar y dar sentido a las acciones que se realizaban con los niños, las niñas y los padres en diferentes proyectos y políticas de intervención lideradas por el ICBF. El interés del ICBF era avanzar hacia la construcción de nuevas formas de relación del niño con los adultos, en todos los escenarios de la vida social.

Por su parte la Unicef, en la primera mitad de la década de los noventa, impartió una serie de pautas de crianza en el contexto familiar, basadas también en las teorías psicológicas del desarrollo infantil. Para este organismo, la concepción que se tenía del niño, las expectativas sociales que se generaban sobre su desempeño, las actitudes y los comportamientos que tipificaban el trato que cada grupo social proporcionaba a sus niños, constituía un elemento fundamental a tener en cuenta en las prácticas crianza. El desarrollo infantil se concebía, para la Unicef, como un proceso determinado, fundamentalmente, por la interacción entre la maduración biológica, la interacción social y las experiencias de aprendizaje. Dicho desarrollo no 
podía ser comprendido ni intervenido fuera del contexto, en el que las condiciones de vida que englobaban la población se convertían en el contexto que comprendía todos los factores de riesgo del desarrollo infantil. De manera específica:

El desarrollo infantil ocurre en interacción y en mediación con otros seres humanos. Esta interacción es cualitativamente diferente en las distintas etapas, ya que el niño es un ser activo que afecta y es afectado por las interacciones con sus padres, su familia y demás miembros de la comunidad... Es preciso que a la maduración biológica que posibilita diferentes y cada vez más complejos niveles de conducta, se añada el ejercicio y actividad del niño en interacción con el medio. Esta interacción no se produce en una sola dirección, no es solamente el niño quien resulta afectado, el entorno que los rodea también es modificado por su actividad $^{13}$.

La Unicef promueve la necesidad de que en el interior de la familia, el niño fuera visto como un ser humano que se desarrolla como totalidad, con sus potencialidades de aprendizaje y desenvolvimiento comportamental, el cual funciona como un sistema compuesto de múltiples

13 Unicef. Programa de Educación Familiar para el Desarrollo Infantil, PEDAFI. Ministerio de Educación. Bogotá, 1993, p. 22. subsistemas: motriz, perceptivo, intelectual y de lenguaje. El reconocimiento del carácter sociocultural del desarrollo infantil y sus factores determinantes son condición necesaria para decidir y orientar adecuadamente las acciones tendientes a prevenir, neutralizar o recuperar posibles desviaciones o retardos con respecto a lo esperado, de acuerdo a la edad y a las condiciones y expectativas culturales del niño.

Para la Unicef se hacía necesario aclararles a la familia y a los educadores que, el desarrollo infantil no era igual a crecimiento. Mientras el crecimiento se describía por cambios en el tamaño, el desarrollo se caracterizaba por cambios en cuanto a la complejidad y a la función:

El desarrollo del niño abarcaba el pasaje de un comportamiento inmaduro a un comportamiento maduro, normas de comportamiento que van de lo simple a lo complejo, así como la evolución del niño, de la dependencia a la autonomía adulta. El desarrollo infantil era un proceso de cambio en el que el niño aprendía a dominar niveles más complejos de movimiento, pensamiento $y$ sentimiento, y a interactuar con personas y objetos en el medio ambiente $^{14}$.

El cuidado infantil debía ser visto como un conjunto de actividades que ayudaban en el desarrollo del

14 Unicef. (2004). El desarrollo de la primera infancia: el desafío y oportunidad. Bogotá 
niño, entre ellas la lactancia materna y la alimentación, el amparo y la supervisión, la prevención de enfermedades y la atención en la enfermedad, así como asegurar la participación del niño en la interacción social y un medio ambiente seguro y estimulante para el juego y la explotación. Los programas de desarrollo para la primera infancia abarcaban iniciativas tanto de cuidado infantil como de educación infantil. Desde la perspectiva de las teorías del desarrollo

Los primeros dos años se caracterizan por un crecimiento rápido y algunas de las experiencias del aprendizaje más importantes de la vida, entre ellas caminar y hablar, afrontar el temor y el placer y descubrirse a sí mismo y a los otros. En ambientes físicos, emocionales y sociales favorables, el desarrollo de importantes estructuras cerebrales apoya la adquisición de habilidades de comportamiento cada vez más complejas que aumentan las capacidades del niño para entablar y aprender las interacciones con las personas que le brindan cuidado, así como con el medio ambiente... La etapa comprendida entre los tres y los seis años de edad también se caracteriza por un desarrollo físico y mental acelerado. Los niños adquieren confianza en sus cuerpos, se esfuerzan por lograr su independencia haciendo cosas por sí mismos y experimentando con objetos en el medio ambiente que los rodea. Muestran una curiosidad más viva por lo que ocurre a su alrededor, disfrutan de la compañía de otros niños y tratan de imitar el comportamiento de los adultos. Aprenden a afirmarse como personas y empiezan a adquirir autocontrol y disciplina. En este periodo el desarrollo intelectual y social del niño progresa rápidamente como lo demuestra la adquisición de habilidades lingüísticas sofisticadas y la adopción de comportamientos aceptables culturalmente. En esta etapa es esencial que a los niños se les asigne tareas que respondan a su intensa sed de aprendizaje y que se les prepare para el pensamiento simbólico y lógico necesario en la educación escolar ${ }^{15}$.

Para la Unicef los niños son física y mentalmente activos y se esfuerzan por entender el mundo. El desarrollo de las 
funciones mentales de orden superior, tales como la conceptualización, empiezan con la interacción social y más tarde se internalizan psicológicamente. El aprendizaje infantil mostraba un ciclo recurrente que empezaba con la toma de conciencia y pasaba luego por la exploración, la indagación y, por último, la utilización. Dentro de los enfoques complementarios a las teorías de desarrollo infantil se encontraba la educación de las personas encargadas del cuidado de los niños y la promoción de los principios del desarrollo dentro de la comunidad.

El ICBF y la Unicef, a lo largo de los años ochenta y noventa se apoyan en la teoría del desarrollo cognitivo para complementar las teorías del desarrollo infantil, en contextos particulares de carácter social y cultural. La familia debía acercarse a las prácticas de crianza en las que se valora a una infancia que sufría una serie de cambios cualitativos, que se transformaba y desarrollaba. Es así como a la primera superficie de emergencia enunciativa en la que en encontramos los enunciados: desarrollo, aprendizaje, esquema, asimilación y acomodación; se agregan otros dos enunciados de carácter fundamental: crianza y socialización. A la anterior serie se sumarían otro grupo de enunciados: atención integral, condiciones de vida, contexto sociocultural, interacción social, cuidado, exploración, indagación, autocontrol, disciplina y toma de conciencia.

\section{Algunas consideraciones finales:}

La teoría del desarrollo cognitivo de Jean Piaget al instalarse en las últimas décadas del siglo XX e inicios XXI, evidencia el desconocimiento que los colombianos teníamos en torno a los temas de infancia, tanto en contextos de carácter formal como la escuela, al igual que en contextos informales como la familia. Sin duda, la teoría del desarrollo cognitivo valorada como una formación discursiva en la cual ubicamos enunciados tan importantes como: esquema, asimilación, acomodación y aprendizaje; acompañados de otros como: socialización y crianza, conllevan a valorar a la infancia como un sujeto cognitivo que vive continuas transformaciones de carácter cualitativo que la sociedad apenas hoy valora.

Las prácticas educativas de carácter pedagógico y escolar, acompañadas de las prácticas de crianza, se transforman de manera sustancial producto de la presencia de la teoría del desarrollo cognitivo de Jean Piaget, las cuales son difundidas en Colombia, por la prensa, como también por el ICBF, el Ministerio de Educación y la Unicef, en el periodo comprendido entre 1968 y 2006.

Los enunciados que acompañan la teoría del desarrollo cognitivo de Jean Piaget, se constituyen en una condición de posibilidad para la emergencia de la infancia en Colombia, para su reconocimiento, objetivación y transformación. 
Otros elementos que debemos tener en cuenta en esta condición de posibilidad histórica para que se constituya la infancia como acontecimiento es la traducción de las obras de Jean Piaget a la lengua castellana, solo hasta la primera mitad de la década de los años setenta, sumado a la masificación del preescolar en Colombia, que se da también para esa misma época. La primera infancia, logra un estatuto importante en la escuela y la sociedad producto de la masificación de la teoría del desarrollo cognitivo y la masificación del preescolar. Finalmente, podríamos decir que la teoría de desarrollo cognitivo representa un elemento consustancial de las demás teorías del desarrollo infantil, desarrollo moral y desarrollo humano en general.

\section{Fuentes}

Diario El Tiempo, 1968-2006.

Unicef-Colombia, ICBF. (1973). Memorias del Primer Seminario Nacional Intersectorial de Alimentación y Nutrición. Comité Nacional de Políticas sobre Alimentación y Nutrición. Palmira, Valle.

ICBF. (1990). El desarrollo infantil, una conceptualización desde el ICBF. Bogotá.

Unicef, Programa de Educación Familiar para el Desarrollo Infantil. Bogotá: PEDAFI, Ministerio de Educación, Unicef.

Unicef. (1994) El desarrollo de la primera infancia: el desafío y oportunidad. Bogotá.

Unicef-ICBF. (1995). Escala de valoración cualitativa del desarrollo infantil, desde el nacimiento hasta los seis años. Bogotá.

Unicef. (1981). Programas de educación infantil temprana en América Latina. Buenos Aires.

\section{Referencias}

Cerda, H. (1986). La institución preescolar. Bogotá: U. Santo Tomas.

Dusel, I. \& Caruso, M. (2006). La invención del aula. Una genealogía de las formas de enseñar. Buenos Aires: Santilla.

Narodowski, M. (1999) Después de clase. Desencantos y desafíos de la escuela actual. Buenos Aires: Novedades Educativas.

Piaget, J. (1973) Psicología y pedagogía. Barcelona: Ariel. (1974) A dónde va la educación. Barcelona: Teide.

Veyne, P. (1984). Foucault revolucionó la historia. En Cómo se escribe la historia, Madrid, Alianza Editorial. 